\title{
A DEMONSTRATION OF THE TRIAL AND ERROR METHOD OF LEARNING.
}

\author{
BY D.ANIEL STARCH, PH.D., \\ University of Wisconsin.
}

Learning by trial and error is undoubtedly the most fundamental method by which the child acquires motor control. The two other methods of learning, ${ }^{1}$ by imitation and by understanding or ideational control, are chiefly supplementary to this primary method. Its importance is too well known to require further comment.

The object of this paper is to describe what has proved to be a convenient laboratory experiment for demonstrating the trial and error method of learning and also to present some typical results obtained thereby.

The experiment consisted of tracing the outline of a six-pointed star as seen in a mirror. This activity is particularly well adapted for demonstrating trial and error because it involves the establishment of new coördinations between motor and perceptual processes. The experiment can also at the same time be used to illustrate crosseducation.

The use of the star pattern has several advantages over other outlines that might be used, as for example, the irregular maze. First, it requires frequent change in the direction of movement, which insures sufficient difficulty and variety of action. Second, the different sections of the outline are of equal length, which furnishes regularity. Third, the outline is of sufficient length to be reasonably difficult and yet not cause noticeable fatigue. The pattern used was three and one half inches in diameter across the extremes of the points.

The experiment as performed by the students is as follows: ${ }^{2}$

${ }^{1}$ Mentioned for example by Kirkpatrick in Fundamentals of Child Study, p. 83 .

${ }^{2}$ A simple and convenient wooden frame used in the experiment for holding the mirror and writing material and shielding the hand from direct view was devised by Prof. W. F. Dearborn. It consists essentially of a horizontal base $18 \times 18$ inches on which the writing material is placed. At the middle of one edge is an upright for holding the mirror. Eight inches above the base and parallel with it is a light wooden board supported at the sides to serve as a shield. 
(a) Tracing one half of one outline with the left hand. (b) Tracing ten complete outlines with the right hand. (c) Tracing another half with the left hand. The results are then tabulated to show the exact time and number of errors of each tracing. The two tracings made with the left hand serve to illustrate the effect of practice with the right hand upon the left hand. Only half of the outline was traced in order not to gain too much practice with the left hand.

The first attempts demonstrate in a convincing way the trial and error procedure. It is brought out particularly well by the several difficult places encountered. A typical illustration is given in Fig. I. In those situations an effort to reason out the direction of movement is of little or no help. Apparently the only way to reach the line is to keep on trying until one succeeds.

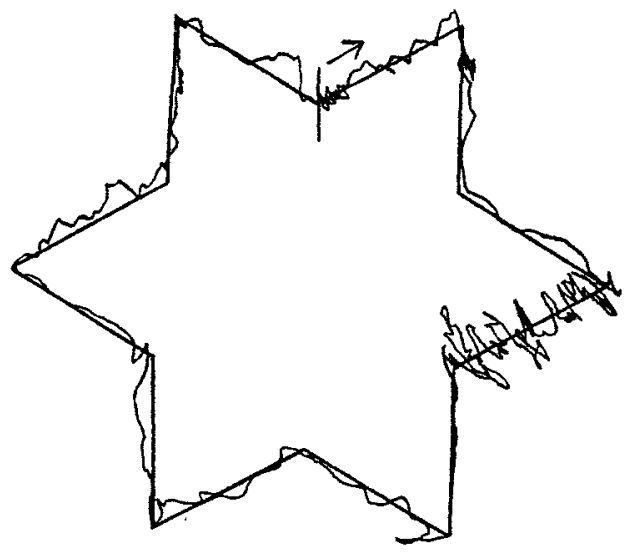

FIG. I.

Fig. 2 presents a typical curve of acquiring the coördinations in the star tracings. It represents one hundred records made by the writer with the right hand at the rate of one a day on consecutive days without interruption except between the fortieth and forty-first records (one day) and between the forty-sixth and forty-seventh records (two days). The upper curve is the time curve and the lower one is the error curve.

The two curves represent the usual course of learning in that they indicate very rapid improvement at first followed by slower progress later. This rapid improvement extends in the error curve over the first seven records, while in the time curve it extends over the first twenty records. In this connection it is of interest to observe that the improvement in time and errors aside from the first seven or eight 
records is at no time parallel. Either the error curve improves rapidly and the time curve remains stationary, or vice versa. The error curve shows a gradual lowering after the first rapid drop until about the fiftieth record. This is accompanied in the time curve by the first rapid drop and then a continuous standstill and even loss until about the fiftieth record. After that the error curve has reached its dead level and the time curve again shows a gradual improvement until about the ninetieth record when both seem to have reached their limits.

The implication seems to be that the plateaus during which there is little or no improvement are an indication of more rapid development in some other aspect of the learning process which is not measured by that particular curve. It seems therefore important in studies of learning to obtain as many different means of measuring progress of learning as possible.

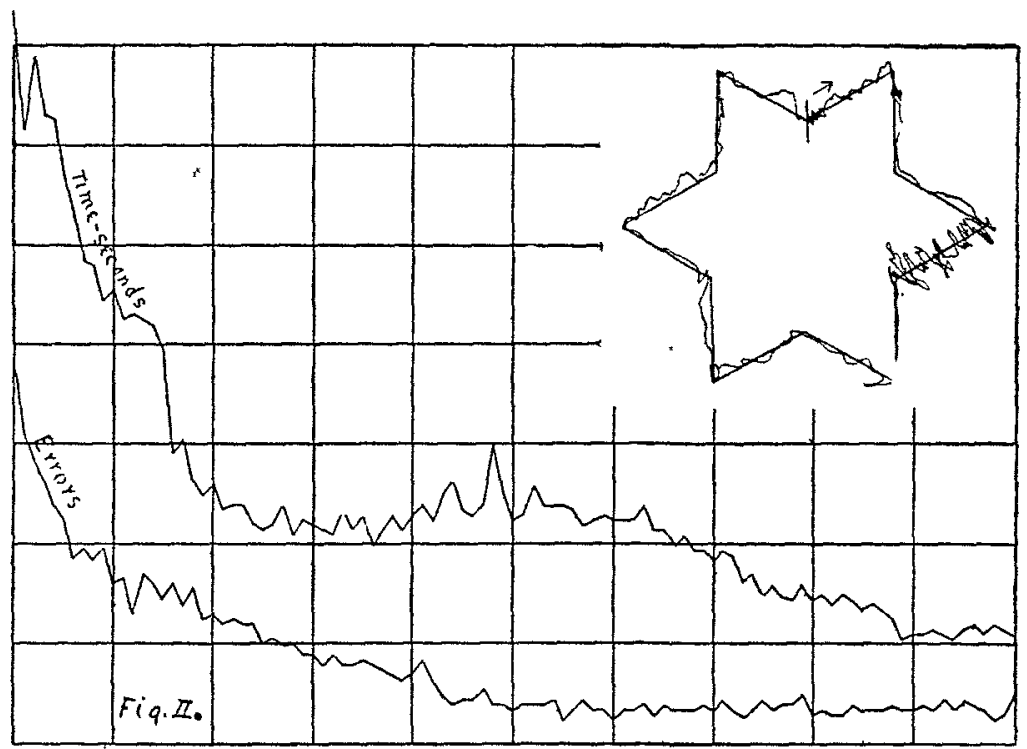

FIG. 2.

The effect of practice with the right hand upon the left hand is very considerable. One tracing was made with the left hand before and one after the practice represented in Fig. 2. The improvement with the right hand from the first to the last record was $\delta_{4}$ per cent. in time and 92 per cent. in errors, average 88 per cent. The improvement of the record made after compared with the one made before the right hand practice was 85 per cent. in time and $8 \mathrm{r}$ per cent. in errors. 
average 83 per cent. Another subject who made fifty tracings with the right hand showed an improvement of 82 per cent., and in the before and after tracings with the left hand 68 per cent. Ten laboratory students who made ten records each with the right hand improved on the average 53 per cent. In the before and after records, tracing with the left hand half of one outline each time, the average improvement was 49 per cent. Taking these results together they show that the left hand profits to the extent of 90 per cent. of the gain made by the right hand. From this however a small amount must be subtracted which is due to the practice derived from the first left hand tracing.

This experiment thus has several commendable points as a laboratory exercise. It is simple and convenient, it satisfactorily demonstrates cross-education and the trial and error method, and its results are definitely measurable. It could also be used advantageously to investigate several problems in the psychology of learning, in particular, the genetic development of muscular coördination, comparing children with adults, adaptation in acquiring a new motor habit, cross-education, transference of training, and the effect of different intervals between records upon the rate of improvement. 